\title{
Research Progress of Jianpi Qushi Powder Combined with Standard Anti Hp Quadruple Therapy in the Treatment of Hp Infectious Gastritis with Spleen Deficiency and Dampness Stagnation
}

\author{
Qiangcai Mai1 ${ }^{*}$, Shoulan Gong2, Guosheng Su${ }^{3 \#, ~ L i h u a ~ Q i n ~}{ }^{3 \#}$ \\ ${ }^{1}$ Guigang Orthopedic Hospital of Integrated Traditional Chinese and Western Medicine, Guigang, China \\ ${ }^{2}$ Guigang Skin Disease Prevention and Treatment Hospital, Guigang, China \\ ${ }^{3}$ People's Hospital of Guangxi ASEAN Economic and Technological Development Zone/Nanning Tenth People's Hospital, Nanning, \\ China \\ Email:18007856271@163.com, "563449581@qq.com
}

How to cite this paper: Mai, Q.C., Gong, S.L., Su, G.S. and Qin, L.H. (2022) Research Progress of Jianpi Qushi Powder Combined with Standard Anti HP Quadruple Therapy in the Treatment of HP Infectious Gastritis with Spleen Deficiency and Dampness Stagnation. Chinese Medicine, 13, 15-21. https://doi.org/10.4236/cm.2022.131002

Received: January 16, 2022

Accepted: February 27, 2022

Published: March 2, 2022

Copyright $\odot 2022$ by author(s) and Scientific Research Publishing Inc. This work is licensed under the Creative Commons Attribution International License (CC BY 4.0).

http://creativecommons.org/licenses/by/4.0/

\begin{abstract}
Stomach Helicobacter pylori infection is one of the common gastric diseases. Helicobacter pylori infectious gastritis of spleen deficiency and dampness stagnation is a relatively intractable chronic disease. In recent years, traditional Chinese medicine methods have emerged one after another in the treatment of gastric Helicobacter pylori infection. By collecting references, the author reviewed the clinical characteristics of gastric Helicobacter pylori infection and the new progress of traditional Chinese medicine treatment.
\end{abstract}

\section{Keywords}

Traditional Chinese Medicine Jianpi Qushi Powder, Quadruple Therapy, Spleen Deficiency Dampness Stagnation Type, Helicobacter Pylori, Infection, Gastritis

\section{Introduction}

Helicobacter pylori $(\mathrm{Hp})$ is an important pathogenic bacterium, which was first discovered in 1982. Research results showed that this bacterium could not only cause gastritis and gastric ulcer, but also was closely related to MALT lymphoma

${ }^{\star}$ Co-first author.

${ }^{\#}$ Co-corresponding author. 
and gastric cancer. It is the only bacterial pathogen that has been confirmed by WHO to be related to human tumorigenesis. Recent research results also found that $\mathrm{Hp}$ also has a high correlation with cardiovascular diseases such as human coronary heart disease. Hp infection is a global problem, and it is estimated that more than $50 \%$ of the global population is infected. The infection rate of $\mathrm{Hp}$ in developed countries is $30 \%-50 \%$, and that in developing countries is about $80 \%$. About one-third of adults in northern Europe and North America have Hp infection, while the prevalence of Helicobacter pylori is usually higher than 50\% in southern and Eastern Europe, South America and Asia. In China, the infection rate of the general population is about $50 \%-80 \%$, and it increases at a rate of $1 \%-2 \%$ every year. A survey of the general population in areas with high incidence of gastric cancer shows that the prevalence of $\mathrm{Hp}$ is 63.4\% [1] [2] [3] [4] [5]. It can be seen that $\mathrm{Hp}$ is one of the pathogenic bacteria with high infection rate, and it has a global epidemic trend; however, there are significant differences in infection rates around the world. Hp can infect people of different ages, and the infection rate varies in different countries and regions due to differences in economic level and living habits. China is one of the countries with high $\mathrm{Hp}$ infection rate and gastric cancer incidence. Hp infection is not only a clinical problem to be solved, but also a major concern of health management. How to formulate a treatment plan for $\mathrm{Hp}$ infection is very important for the prevention and treatment of Hp-related diseases in China. The literature is now collected and reviewed as follows.

\section{Research Status of Helicobacter pylori Infection at Home and Abroad}

Since the discovery of Helicobacter pylori $(\mathrm{Hp})$, a large number of studies at home and abroad have shown [6] [7] [8] that Helicobacter pylori is the main cause of chronic active gastritis and peptic ulcer infection. Helicobacter pylori infection is also associated with gastric lymphoma, gastric cancer and dyspepsia. The World Health Organization Agency for Research on Cancer (IARC) defined Helicobacter pylori as a class I carcinogen in 1994. Multinational health departments take Helicobacter pylori eradication as a guideline for preventing gastric cancer.

Helicobacter pylori infection is a global problem [9] [10], and it is estimated that more than $50 \%$ of the global population is infected. Hp infection rate is $30 \%$ - $50 \%$ in developed countries and about $80 \%$ in developing countries. About one third of adults in northern Europe and North America have Hp infection, while the prevalence of Helicobacter pylori is usually higher than $50 \%$ in southern and Eastern Europe, South America and Asia. In China, a survey of the general population in areas with high incidence of gastric cancer showed that the prevalence of $\mathrm{Hp}$ was $63.4 \%$. It can be seen that $\mathrm{Hp}$ is one of the pathogenic bacteria with high infection rate and shows a global epidemic trend, while there are significant differences in infection rates all over the world. 


\section{Clinical Diagnosis and Treatment Mechanism of Gastric Helicobacter pylori Infection at Home and Abroad}

A large number of studies and clinical treatment practices at home and abroad show that [11] [12] [13] [14] [15] Hp infection and colonization is the primary condition for its pathogenesis. If $\mathrm{Hp}$ is removed, gastritis and gastric ulcer will be improved. The $\mathrm{pH}$ value of membrane layer in normal human stomach is about $2-4$, and the $\mathrm{pH}$ value of gastric juice is about 2 . Studies by weeks and others have shown that $\mathrm{Hp}$ extracellular urease is inactivated when $\mathrm{pH}$ value is $\leq 4.5$ and survives for less than 5 minutes when $\mathrm{pH}$ value is lower than 4.0. Then why can Hp survive in a highly acidic environment in the stomach? Weeks and Scott et al. believed that Hp urea channel ure I could absorb urea from the extracellular, and contribute to the decomposition of intracellular urease into ammonia $\left(\mathrm{NH}_{3}\right)$ and carbon dioxide $\left(\mathrm{CO}_{2}\right)$. The "ammonia cloud" formed by ammonia $\left(\mathrm{NH}_{3}\right)$ created a "comfortable" environment with low oxygen and weak acid for Hp colonization, which was the necessary molecule for Hp colonization. However, the immunological properties of ureI and whether it can be used as a drug target to prevent $\mathrm{Hp}$ infection have not been further reported.

At present, from the current situation of clinical treatment of Hp, the conventional treatment of $\mathrm{Hp}$ infection is the use of antibiotics or combined antibiotics. Although this medical measure of broad-spectrum antibiotic treatment has a certain effect on the individual elimination of $\mathrm{Hp}$, this treatment is easy to cause flora disorder and the production of drug-resistant strains in the human body, and is not conducive to the prevention and treatment of $\mathrm{Hp}$ in the stomach and natural environment. Although PPI (Proton Pump Inhibitor) treatment can inhibit gastric acid secretion, as the $\mathrm{pH}$ value of gastric environment rises; it creates a neutral environment for $\mathrm{Hp}$ colonization in the stomach, which is conducive to the increase of $\mathrm{Hp}$ quantity. Starting from the molecular role and biochemical mechanism involved in the physiological metabolism of $\mathrm{Hp}$, the immune technology is used to block the urea membrane channel of Hp, so as to block the biochemical reaction of urease decomposing urea. At the same time, the urease B subunit of $\mathrm{Hp}$ is used as the target to eliminate the activity of decomposing urea in the extracellular cells of $\mathrm{Hp}$, so that $\mathrm{Hp}$ cannot resist the acid environment of the stomach and lead to death or non-colonization. This multi-target fusion design using the key molecules of $\mathrm{Hp}$ colonization achieves the purpose of both preventing and treating Hp infection, and avoids the clinical shortcomings of antibiotics and PPI reagents in the prevention and treatment of $\mathrm{Hp}$; this combined application of multi gene and multi-target dominant antigen epitopes is an ideal method for the prevention and treatment of Hp [16] [17] [18] [19] [20]. Clinically, standard bismuth quadruple therapy [21] [22] [23], standard quadruple therapy: Pantoprazole capsules $40 \mathrm{mg}$ Qd, amoxicillin capsules $1.0 \mathrm{~g}$, Bid, clarithromycin tablets $0.5 \mathrm{~g}$ Bid, bismuth potassium citrate tablets $0.6 \mathrm{~g}$ Bid, 14 days as a course of treatment. At the same time, breath test and gastroscopy are performed in the two groups before treatment and 4 weeks after 
treatment, and the clinical symptom score, ulcer area, efficacy evaluation and incidence of adverse reactions are statistically analyzed.

\section{Current Status of Traditional Chinese Medicine Treatment of Gastric Helicobacter pylori Infection}

According to Chinese medicine, Helicobacter pylori belongs to the category of "evil qi", "where the evil qi gathers, its qi must be deficient", "the righteous qi is stored, the evil cannot dry", and strengthening the righteousness and eliminating the evil is the basic principle for the treatment of Helicobacter pylori-related diseases. According to the method of dividing treatment of deficiency and excess, those with excess can reduce it, those with deficiency can supplement it, and those with both deficiency and excess can supplement and reduce it. The excess is mainly manifested as dampness and heat, and the method of dispelling evil focuses on clearing heat and dispelling dampness. Deficiency is mainly manifested as spleen deficiency, and the method of strengthening health focuses on tonifying middle Qi, strengthening spleen and stomach."Syndrome" is not only a bridge between the basis of traditional Chinese medicine and clinical treatment, but also an important link in the treatment of traditional Chinese medicine.

Syndrome differentiation and treatment is an individualized treatment based on personal signs, that is, according to the characteristics of tongue coating, symptoms, signs and pulse of each patient, use the four diagnosis and reference to determine the disease type of traditional Chinese medicine, and then give different prescriptions for treatment according to different diseases.

The latest treatment of $\mathrm{Hp}$ infection mainly refers to the rational application of non antibiotic drugs such as mucosal protectants, probiotics and traditional Chinese medicine in the treatment of Hp infection related diseases. Studies have shown that [24] [25], traditional Chinese medicine has a good effect in the treatment of $\mathrm{Hp}$ infection related diseases, and has a broad prospect of clinical application. According to the current clinical research, the drugs with good effect on the treatment of $\mathrm{Hp}$ infection related diseases include Wenweishu, Jinghuaweikang, Yangweishu, moluodan, Weifuchun, etc. These traditional Chinese medicines not only have good clinical efficacy, but also some probiotics such as lactobacillus, Saccharomyces braldii, as well as mucosal protectants combined with standard triple or quadruple combination including antibiotics can improve the eradication rate of $\mathrm{Hp}$ and reduce adverse reactions during treatment. The effectiveness and mechanism of traditional Chinese medicine in the treatment of $\mathrm{Hp}$ infection need more in-depth and meticulous confirmation by more researchers through basic and clinical research. At present, the treatment method of traditional Chinese medicine is mainly through systemic regulation to achieve the therapeutic effect, which can directly inhibit and kill Hp. Traditional Chinese medicine treatment can improve the clinical symptoms and quality of life of patients with $\mathrm{Hp}$ infection. 


\section{Summary and Prospect}

In conclusion, with the extensive drug treatment of $\mathrm{Hp}$ infection, drug resistance is increasing, and the probability of eradicating $\mathrm{Hp}$ is also decreasing year by year. The treatment of Helicobacter pylori infection is facing great challenges. In recent years, the treatment plan of Helicobacter pylori has changed from tripartite combination to quadripartite combination, and the course of treatment has been extended continuously. The dosage of antibiotics has been increasing, but the efficacy is very limited, and the side effects have been increasing, and a few patients have repeated attacks. The treatment of $\mathrm{Hp}$ has entered a difficult period. Exploring the treatment of $\mathrm{Hp}$ infection with Traditional Chinese medicine has become a new idea for many domestic researchers. In recent years, some scholars and research institutions in China have also studied the treatment plan of traditional Chinese medicine, and have achieved certain breakthroughs with exact curative effects, which have been well received by the majority of patients. In order to understand the therapeutic effect of Traditional Chinese medicine Jianpi Qushi powder on Hp infectious gastritis of spleen deficiency and dampness stagnation type, this study used Traditional Chinese medicine Jianpi Qushi powder combined with standard anti Hp quadruple therapy to explore and study the treatment of $\mathrm{Hp}$ infectious gastritis of spleen deficiency and dampness stagnation type, hoping to achieve better research results.

\section{Acknowledgements}

During the process of this topic research, we got much help from many departments and individuals, and other personnel not involved in this project research. All of them offered a great support and help in this research. Now here, all of members in this research show our deepest appreciation to them, and wish them good health and everything goes well.

\section{Funding}

Scientific Research Project Funded by the Bureau of Traditional Chinese Medicine of Guangxi Zhuang Autonomous Region (No.: gzzc2019157).

\section{Conflicts of Interest}

The authors declare no conflicts of interest regarding the publication of this paper.

\section{References}

[1] Kimura, K., Taniguchi, Y., Satoh, K., et al. (1997) Composition for the Treatment of Helicobacter pylori Infection. Kaken Pharmaceutical Co Ltd., US5618564A.

[2] Technische Universität München and Max-Planck-Gesellschaft zur Förderung der Wissenschaften E.V. (2017) Helicobacter pylori Vaccines. World Patent Organization, WO2017102779A1.

[3] Abadi, A. (2015) Helicobacter pylori and Gastric Cancer: Clinical Aspects. Chinese 
Journal of Medicine (English Version), 128, 3101-3105. https://doi.org/10.4103/0366-6999.169107

[4] Yang, J.C., Shun, C.T., Chien, C.T. and Wang T.H. (2010) Composition for the Prevention and the Treatment of Helicobacter pylori Infection. National Taiwan University, US2010298244A1.

[5] Shiota, S. and Yamaoka, Y. (2014) Strategy for the Treatment of Helicobacter pylori Infection. Current Pharmaceutical Design, 20, 4489-4500.

[6] Garault, P., Bourdet-Sicard, R. and Megraud, F. (2014) Streptococcus thermophilus Strains for Treating Helicobacter pylori Infection. World Patent Organization, WO2014064488A1.

[7] SHIONOGI \& Co Ltd. (2000) Vaccine Composition for Infection of Helicobacter pylori. Shionogi \& Co Ltd., JP2000083671.

[8] Harris, A. (2001) Treatment of Helicobacter pylori. World Journal of Gastroenterology, 7, 303-307.

[9] CHIRON Corporation. (1998) Helicobacter pylori Diagnostics. Chiron Corporation, CA2270163A1.

[10] Jonsson, A.B. and Wehelie, R. (2011) Compounds, Medicaments and Methods of Treatment for Helicobacter pylori Infection. World Patent Organization, WO2011139226A1.

[11] Jonsson, A.B. and Wehelie, R. (2013) Compounds, Medicaments and Methods of Treatment for Helicobacter pylori Infection. European Patent Office, EP2566498A1.

[12] Go, M.F. and Fennerty, M.B. (1998) Treatment of Helicobacter pylori Infection. Current Opinion in Gastroenterology, 14, 64-69.

[13] Coton, T., Debonne, J.M., Guisset, M., et al. (1997) L'infection a Helicobacter pylori dans les pays en developpement. Medecine Tropicale, 57, 77-82.

[14] Lin, C.H. and Liu, T.W. (2014) Compositions and Assays for Treatment and Diagnosis of Helicobacter pylori Infection and Conditions. US8785402B2.

[15] Chey, W.D., Leontiadis, G.I., Howden, C.W., et al. (2017) ACG Clinical Guideline: Treatment of Helicobacter pylori Infection. The American Journal of Gastroenterology, 112, 212-239.

[16] O’Connor, A., O'Morain, C.A. and Ford, A.C. (2017) Population Screening and Treatment of Helicobacter pylori Infection. Nature Reviews Gastroenterology \& Hepatology, 14, 230-240.

[17] RedHill Biopharma Ltd. (2017) Pharmaceutical Compositions for the Treatment of Helicobacter pylori. Red Hill Biopharma Ltd., US2017189341A1.

[18] Tongtawee, T., Wattanawongdon, W. and Simawaranon, T. (2019) Effects of Periodontal Therapy on Eradication and Recurrence of Helicobacter pylori Infection after Successful Treatment. Journal of International Medical Research, 47, 875-883.

[19] Ranjbar, R., Behzadi, P. and Farshad, S. (2017) Advances in Diagnosis and Treatment of Helicobacter pylori Infection. Acta Microbiologica et Immunologica Hungarica: A Quarterly of the Hungarian Academy of Sciences, 64, 273-292.

[20] Dong, H., Jin, S.L. and Miao, D.L. (2017) Interpretation of ACG Clinical Guideline: Treatment of Helicobacter pylori Infection (Version 2017). International Journal of Translational Medicine, 5, 160-166.

[21] Okuda, M., Kikuchi, S., Mabe, K., et al. (2017) Nationwide Survey of Helicobacter pylori Treatment for Children and Adolescents in Japan. Pediatrics International, 59, 57-61. 
[22] Zhang, Y., Sun, H., Zhao, H.L., et al. (2017) Early Apoptosis of Monocytes Induced by Helicobacter pylori Infection through Multiple Pathways. Developmental \& Comparative Immunology, 73, 46-51.

[23] Ribichini, D., Castelli, V., Pasquali, R., et al. (2017) Tablet and Oral Liquid L-Thyroxine Formulation in the Treatment of Naïve Hypothyroid Patients with $\mathrm{He}$ licobacter pylori Infection. Endocrine, 57, 394-401.

[24] Ma, H., Gao, H., Huang, Y.B., et al. (2017) Efficacy Observation of Compound Lactobacillus acidophilus Combined with Conventional Quadruple Therapy in the Treatment of Peptic Ulcer Infected with Helicobacter pylori. Chinese Journal of Hospital Infectious Diseases, 27, 2932-2934, 2946.

[25] Cui, C.C., Li, C.F. and Zhang, B. (2017) Research Status and Progress of Treatment Regimens for Helicobacter pylori Infection. Journal of Jilin University (Medical Edition), 43, 1287-1290. 